\title{
Advances in the role of music in neurorehabilitation: Addressing critical gaps in clinical applications
}

Twenty five years ago, the use of music-based interventions to play a core role in neurorehabilitation besides the adjunctive benefits of psychosocial support was virtually unknown. Since then breakthrough research in clinical applications, followed by studies elucidating underlying brain mechanisms, have established an increasing awareness and recognition of music as a therapeutic modality. As an example, Rhythmic Auditory Stimulation has been adopted since 2019 in the US (VA, DoD) and Canadian (Heart and Stroke) official Stroke Care Guidelines. Starting with new research in the $1990 \mathrm{~s}$ showing for the first time how musical-rhythmic stimuli can improve mobility in stroke and Parkinson's disease to revisiting the multiple connections between music and the language and speech functions to being able to demonstrate that music-based interventions can shape and re-train cognitive functions such as attention, memory or the executive, we are at a point where we can also state clinically that the brain that engages in music is changed by engaging in music.

This thematic issue of NeuroRehabilitation is intended to contribute new knowledge in clinical domains and populations less researched so far. This issue includes three studies on music in traumatic brain injury rehabilitation, which is a highly underresearched area. The sample sizes are small from case studies to a small Randomized Controlled Trial but the results provide an important basis of feasibility for future research. Two studies look at music-based interventions in children, another under-researched group, with autism and learning disabilities respectively. An important little investigated connection between motor training and cognitive outcomes in chronic stroke rehabilitation is addressed in another paper. Finally, a theoretical paper on the mechanisms on neuroplastic changes underlying successful Neurologic Music Therapy interventions will provide a theoretical understanding of how music shapes brain function in neurorehabilitation on an impairment level. We hope that this thematic issue can begin to fill in some critical gaps of knowledge in less researched applications and thus contribute to the advancement of music in brain rehabilitation.

Michael H. Thaut, PhD Professor of Music Professor of Neuroscience (IMS, CPIN) and Rehabilitation Science (RSI)

Director, Music and Health Science Research Collaboratory

Director, Graduate Programs in Music and Health

Sciences

Collaborator Scientist CAMH Neuroimaging Affiliate Scientist St. Michael's Hospital Canada Research Chair: Tier 1 University of Toronto E-mail: Michael.thaut@utoronto.ca Tel.: +1 416-978-0535 Fax : +1 416-946-3353 